FTPI-MINN-11/18

UMN-TH-3008/11

\title{
Decomposing Instantons in Two Dimensions
}

\author{
Muneto Nitta $^{1}$ And Walter Vinci ${ }^{2}$ \\ ${ }^{1}$ Department of Physics, and Research and Education Center for Natural Sciences, \\ Keio University, 4-1-1 Hiyoshi, Yokohama, Kanagawa 223-8521, Japan \\ ${ }^{2}$ University of Minnesota, School of Physics and Astronomy \\ 116 Church Street S.E. Minneapolis, MN 55455, USA \\ nitta(at)phys-h.keio.ac.jp \\ vinci(at)physics.umn.edu
}

\begin{abstract}
We study BPS vortices in the $1+1$ dimensional $\mathcal{N}=(2,2)$ supersymmetric $U(1)$ gauged $\mathbb{C} P^{1}$ non-linear sigma model. We use the moduli matrix approach to analytically construct the moduli space of solutions and solve numerically the BPS equations. We identify two topologically inequivalent types of magnetic vortices, which we call $\mathrm{S}$ and $\mathrm{N}$ vortices. Moreover we discuss their relation to instantons (lumps) present in the un-gauged case. In particular, we describe how a lump is split into a couple of component S-N vortices after gauging. We extend this analysis to the case of the extended Abelian Higgs model with two flavors, which is known to admit semi-local vortices. After gauging the relative phase between fields, semi-local vortices are also split into component vortices.

We discuss interesting applications of this simple set-up. Firstly, the gauging of non-linear sigma models reveals a semiclassical "partonic" nature of instantons in 1+1 dimensions. Secondly, weak gauging provides for a new interesting regularization of the metric of semi-local vortices.
\end{abstract}




\section{Introduction}

Instantons play an important role in quantum field theories in various dimensions. In four dimensions, they play a prominent role in defining the properties of the QCD vacuum, and in particular in explaining strong coupling effects like chiral symmetry breaking. In the case of $\mathcal{N}=2$ supersymmetric QCD, they give non-perturbative corrections to the exact low-energy effective action [1]. It has been long pointed out that there are similarities between four dimensional gauge theories and two dimensional non-linear sigma models, such as dynamical generation of a mass gap and asymptotic freedom. These similarities extend to the quantitative level in the supersymmetric case, where it happens that the exact BPS spectra of super QCD and non-linear sigma models are the same [2]. It is not a coincidence that instantons were found in the $O(3)$ sigma model $[3]$ almost at the same time of the discovery of instantons in Yang-Mills theory [4]. The $O(3)$ sigma model is equivalent to $\mathbb{C} P^{1}(\simeq S U(2) / U(1))$ sigma model and therefore instanton solutions can be extended to $\mathbb{C} P^{N-1}(\simeq S U(N) /[S U(N-1) \times U(1)])$ model. In more general terms, when $\pi_{2}(M)$ is nontrivial for the target space $M$, the model admits instantons. Instantons in both YangMills theory and sigma models have a scale modulus in addition to orientational moduli in the internal space and position moduli. In particular, the moduli space of instantons in four dimensional Yang-Mills has real dimension $4 N$, while that of two dimensional sigma model instantons is $2 N$. Sigma model instantons are particle-like object in $2+1$ dimensions, and they are called lumps in field theory [5], and skyrmions or coreless vortices in condensed matter physics. The $\mathbb{C} P^{1}$ model can be lifted to a $U(1)$ gauge theory with two complex scalar fields with equal charges, which reduces to the $\mathbb{C} P^{1}$ model in the limit of gauge coupling sent to infinity. The sigma model instanton is lifted to a semi-local vortex [6 10], having the same moduli including a size modulus.

The moduli space dimension of four and two dimensional instantons, together with other circumstantial observations, has led to the conjecture that instantons can be more conveniently thought as being formed of $N$ component "partons". The moduli space parameters then describe the positions of these fundamental objects in the euclidean space. The idea that the functional integral of strongly coupled Yang Mills is dominated by a liquid of partons which forms the vacuum, explains many aspects of confinement and chiral symmetry breaking [11]. The decomposition of sigma model instantons has been considered also more recently [12,13] and it can occur by deforming the metric of the target space of sigma models. The energy density of the configuration has then subpeaks which can then be interpreted as partons. The proposal of Ref. [12] is to identify these component partons as the UV degrees of freedom which may render $2+1$ sigma models and $4+1$ gauge theories renormalizable. In this paper we discuss, at the semiclassical level, the decomposition of instantons in a manner different from $[12 \sqrt{14}]$. To this end, we consider a two dimensional $\mathcal{N}=(2,2)$ supersymmetric $\mathbb{C} P^{1}$ non-linear sigma model. We then gauge a $U(1)$ subgroup of the full $S U(2)$ isometry enjoyed by the $\mathbb{C} P^{1}$ model. The $U(1)$ gauged $\mathbb{C} P^{1}$ model, is known to admit two Abelian vortices (Abrikosov-Nielsen-Olesen vortices [15]) [16 18]. We show how an instanton is decomposed into these vortices.

The supersymmetric gauged $\mathbb{C} P^{1}$ model admits a unique supersymmetric vacuum up to the gauge symmetry, while the vacuum manifold is a $U(1)$ gauge orbit, a circle, on $S^{2}$. The potential, completely fixed by supersymmetry, has two local maxima at the north and south 

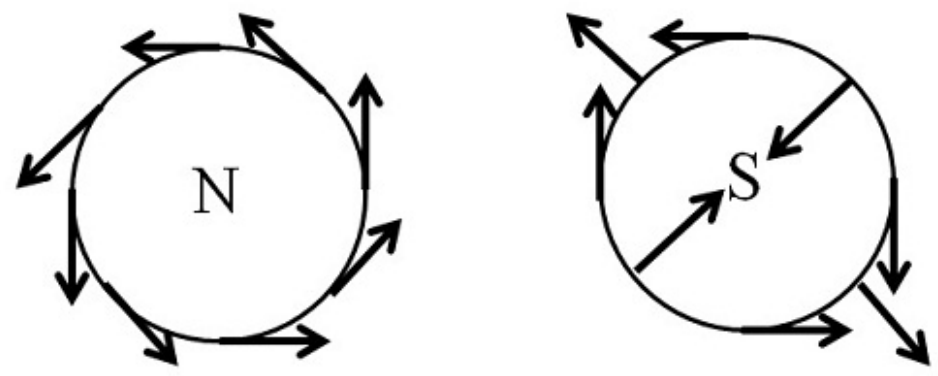

Figure 1: $\mathrm{N}$ and $\mathrm{S}$ vortices. The boundary conditions of the $\mathrm{N}$ and $\mathrm{S}$ vortices are shown by arrows which represent points on the gauge orbit (on the equator) of $S^{2}$. N and $\mathrm{S}$ denote the north and south poles in the vortex cores.

poles. Encircling the outside of a vortex core at the boundary, the $U(1)$ phase winds once around the gauge orbit. When this winding is unwound at the core of a vortex, there exists two choices, the north and south poles, see Fig. 1. Therefore there exist two kinds of vortices with different cores. We call them N and $\mathrm{S}$ vortices in this paper. One instanton wraps $S^{2}$ once while $\mathrm{N}$ and $\mathrm{S}$ vortices wrap upper and lower hemispheres bounded by the vacuum $U(1)$ gauge orbit, see Fig. 2. Accordingly, each of them has a half (or more generally fractional) instanton charge of $\pi_{2}(M)$, so that they can be called two-dimensional merons [19], in analogy with merons in four dimensions [20]. A set of $\mathrm{N}$ and $\mathrm{S}$ vortices can be interpreted as one instanton as can be seen in Fig. 2, where the distance between them corresponds to the size of the instanton. In this sense $\mathrm{N}$ and $\mathrm{S}$ vortices are constituents of an instanton. It was found that the moduli space metric is incomplete at the point where the positions of $\mathrm{N}$ and $\mathrm{S}$ vortices coincide [17]. In our understanding, this is nothing but a small instanton singularity.

Once the $\mathbb{C} P^{1}$ model is constructed as the low energy limit of a linear $U(1)$ gauge theory, the $U(1)$ gauged $\mathbb{C} P^{1}$ model can be formulated as $U(1) \times U(1)$ gauge theory with two complex scalar fields. In this way, the moduli space of instantons is promoted to that of semi-local vortices, which is regular. Then, the small instanton singularities are resolved. No pathology occurs when $\mathrm{N}$ and $\mathrm{S}$ vortices coincide, so that the moduli space is regularized.

Another advantage in considering this $U(1) \times U(1)$ linear formulation is that we can obtain a rather interesting regularization of the semi-local vortex metric. An important recent development concerning the aforementioned correspondence between BPS spectra in two and four dimensions regards the role played by non-Abelian semi-local vortices. More precisely, the correspondence holds between four dimensional $U(N)$ super QCD with $N_{f}>N$ flavors and the two dimensional effective theory on the vortex world-sheet [21,22] hosted by the theory when put on the Higgs phase. This correspondence has been proved using D-brane constructions of the vortex theory. However, while string theory gives a well-defined effective theory, it is well known that some zero-modes are non-normalizable when the effective theory is derived from field theory [23,24]. It is then difficult to quantize the vortex theory and check the correspondence in a fully field theoretic framework. A possible approach to the problem has been considered for example in Ref. 25]. Here we propose weak gauging as an alternative 


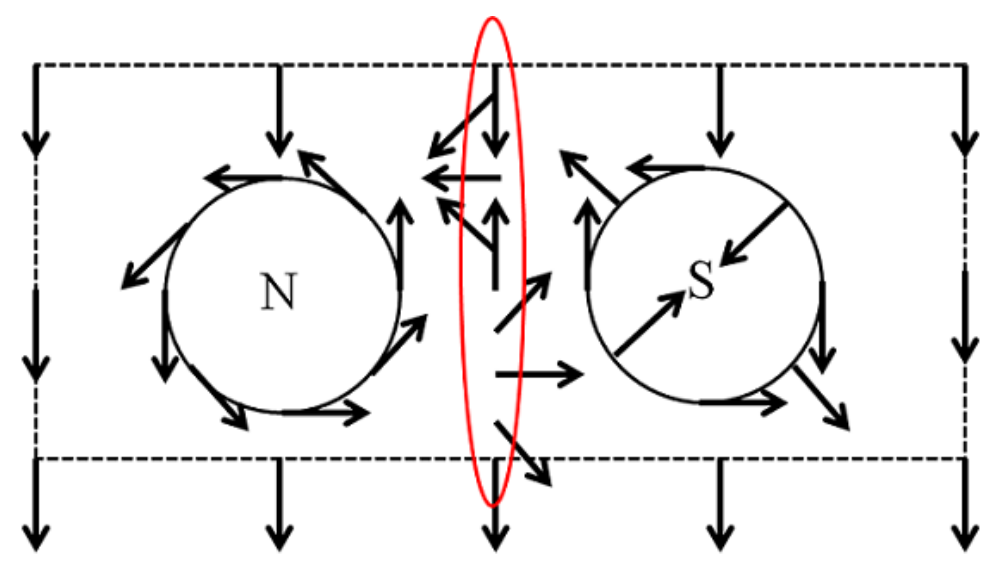

Figure 2: The decomposition of a lump into $\mathrm{N}$ and $\mathrm{S}$ vortices. The whole configuration is a lump with charge one, where the arrows represent points on $S^{2}$. Configurations of $\mathrm{N}$ and $\mathrm{S}$ vortices appear. A gauge orbit exists between them (around the oval region), and it separates the $\mathrm{N}$ and $\mathrm{S}$ vortices.

approach to the problem. The weak gauging of a non-linear sigma model, when considered as the effective theory of a semi-local vortex string, should correspond to a deformation of the four dimensional bulk theory. This would give new $4 \mathrm{~d} / 2 \mathrm{~d}$ correspondences together with important insights on the physics of non-normalizable modes in the undeformed case.

While we have considered only the supersymmetric case, we can also use our analysis to obtain qualitative answers about non-supersymmetric cases. A more generic potential would introduce for example some non-trivial interactions between the component vortices. There exist examples of analogs of these non supersymmetric vortices in condensed matter systems, in which also one or both of the $U(1)$ symmetries are global. The case when both $U(1)$ s are global is relevant in the case of anti-ferromagnets and two component BoseEinstein condensates of ultracold atoms in the anti-ferromagnetic phase [26]. Even if these systems have a global symmetry differing from the supersymmetric gauged $\mathbb{C} P^{1}$ model, they have similar potential terms. Consequently topological properties, for instance whether and how instantons are decomposed into constituent vortices, are the same. Another condensed matter example which is similar to our model is given by two-band superconductors. The Landau-Ginzburg Lagrangian proposed to describe them consists of two gaps (complex scalar fields) coupled to the electro-magnetic $U(1)$ gauge field. It has $U(1) \times U(1)$ symmetry, one of which is local electro-magnetic $U(1)$ symmetry while the other $U(1)$ symmetry, a relative phase between the two fields, is a global $U(1)$ which is broken explicitly in the presence of Josephson interactions between two gaps. This model is also known to admit fractional vortices [27] which are mostly the same with ours in the absence of Josephson interactions ${ }^{1}$. Study of our supersymmetric gauged $\mathbb{C} P^{1}$ model will give insights to these condensed matter systems.

The structure of the paper is the following. In Section 2 we briefly review the construction

${ }^{1}$ However in the presence of Josephson interactions two kinds of vortices are connected by a domain wall $[28$. 
of two-dimensional instantons as $\mathbb{C} P^{1}$ lumps. We then employ the moduli matrix formalism to construct vortices in the gauged version of the sigma model. In Section 3 we construct a bound state of $\mathrm{N}$ and $\mathrm{S}$ vortices and show how they emerge from an instanton configuration as we increase the strength of the gauge interactions. In Section 4 we lift the $\mathbb{C} P^{1}$ non-linear sigma model to a $U(1) \times U(1)$ linear formulation and consider regularization of small instanton singularities. In Section 5 we discuss how the gauging of flavor symmetries can provide for a nice regularization of the semi-local vortex metric. Finally, in Section 6 we present various possible generalizations where we consider sigma models with higher dimensional target spaces and gauging of flavor symmetries of higher rank.

\section{Solitons in Gauged $\mathbb{C} P^{1}$ non-Linear Sigma Model}

\subsection{Models}

\section{Un-gauged $\mathbb{C} P^{1}$}

Let us start by considering the standard two-dimensional $\mathbb{C} P^{1}$ non-linear sigma model $(\mathrm{NL} \sigma \mathrm{M})$. The action is the following [3]:

$$
\mathcal{L}=\xi \frac{\left|\partial_{\mu} b\right|^{2}}{\left(1+|b|^{2}\right)^{2}}, \quad \mu=1,2
$$

In the formula above, $b$ is the holomorphic coordinate parameterizing the target space, and we work with the euclidean metric. The metric for $b$ is given by the standard round Fubini-Study metric and it is written in a patch which does not include the point at infinity $b=\infty$. We can include this point performing a change of variable $b=1 / b^{\prime}$, under which the lagrangian (2.1) is indeed invariant. The standard Fubini-Study metric is of the Kähler type, since it can be derived from a Kähler potential:

$$
\mathcal{L}=\partial_{b} \partial_{\bar{b}} K(b, \bar{b})\left|\partial_{\mu} b\right|^{2}, \quad K=\xi \log \left(1+|b|^{2}\right) .
$$

The Kähler property of the metric ensures the existence of a supersymmetric extension with four supercharges of the bosonic NL $\sigma \mathrm{M}[29]$. The analyses of this paper can be extended to a study of static string-like solutions with translational symmetry in $3+1$ dimensions. Formula 2.1) will then represent the dimensionally reduced four-dimensional action to the plane transverse to the string. From the point of view of constructing solitons, the model we consider can be considered as either being a truncated bosonic sector of an $\mathcal{N}=2$ theory in four dimensions or an $\mathcal{N}=(2,2)$ theory in two dimensions. Since our analysis is limited to the classical level, we never show explicitly the fermionic sector. Supersymmetry however fixes the kinetic terms and the potentials we will consider later.

Since the $\pi_{2}$ of the target space is nontrivial, the $\mathbb{C} P^{1} \mathrm{NL} \sigma \mathrm{M}$ contains stable topological solitons of codimension two. This means string-like objects in four dimensions or instantons in two euclidean dimensions. Solitons can be found with a square root completion of the Bogomol'nyi type [3]. If we restrict ourselves to consider static solutions, we can rewrite the 
lagrangian 2.1 in the following way

$$
\begin{aligned}
\mathcal{E} & =2 \xi \frac{\left|\partial_{z} b\right|^{2}+\left|\partial_{\bar{z}} b\right|^{2}}{\left(1+|b|^{2}\right)^{2}}=2 \pi \xi N+4 \xi \frac{\left|\partial_{\bar{z}} b\right|^{2}}{\left(1+|b|^{2}\right)^{2}}, \\
z & \equiv x_{1}+i x_{2} \quad \partial_{z} \equiv \frac{1}{2}\left(\partial_{1}-i \partial_{2}\right) .
\end{aligned}
$$

The quantity

$$
N \equiv \frac{1}{\pi} \frac{\left|\partial_{z} b\right|^{2}-\left|\partial_{\bar{z}} b\right|^{2}}{\left(1+|b|^{2}\right)^{2}}
$$

gives the degree of the map $b(z): \mathbb{C} P^{1} \rightarrow \mathbb{C} P^{1}$ and is the topological integer which characterizes the second homotopy group:

$$
\pi_{2}\left(\mathbb{C} P^{1}\right)=\mathbb{Z}
$$

Clearly the energy has a lower bound $E \geq 2 \pi \xi N$ which is saturated when the following equation is satisfied:

$$
\partial_{\bar{z}} b=0 \text {. }
$$

Topological solitons of the type above are BPS (Bogomol'nyi-Prasad-Sommerfeld) saturated [30, 31]. They satisfy first order equations of motion obtained from a square root completion and their energy is proportional to a topological integer. In theories with extended supersymmetry, the mass of BPS solitons saturates the bound given by a central extension of the supersymmetry algebra [32]. In the language of supersymmetry, lumps are $1 / 2 \mathrm{BPS}$, in the sense that they preserve $1 / 2$ of the supersymmetry transformations [33. Fermions and supersymmetry are crucial to preserve BPS saturation once quantum effects are taken into account [34].

Since the energy is proportional to the topological integer $N$, which also counts the number of solitons, there are no static interactions among lumps. The consequence of this fact is the existence of a large set of degenerate solutions (moduli space) parameterized by the positions and orientation of the single component lumps. This degeneration can be understood easily from a mathematical point of view if we notice that the equation above implies that $b$ is a holomorphic function of the complex variable $z$. It must contain a finite number of poles and zeroes, and must then be given by a holomorphic rational function [3].

$$
b(z)=b_{\infty} \frac{z^{N}+p_{1}^{N-1}+\cdots+p_{N}}{z^{N}+q_{1}^{N-1}+\cdots+q_{N}}, \quad b_{\infty} \equiv b(\infty)
$$

where the degree $N$ of the polynomials is the lump number (2.4). A fundamental lump is given for example by the following choice:

$$
b_{0}(z)=b_{\infty} \frac{z-z_{S}}{z-z_{N}} .
$$


To extract physical quantities such as size and position from the rational map above, we can make use of the $S U(2)$ isometry enjoyed by the $\mathbb{C} P^{1} \mathrm{NL} \sigma \mathrm{M}$ which acts non-linearly on the field $b$ :

$$
b \rightarrow \frac{v+u b}{u^{*}-v^{*} b}, \quad\left(\begin{array}{cc}
u^{*} & -v^{*} \\
v & u
\end{array}\right) \in S U(2), \quad|u|^{2}+\left|v^{2}\right|=1 .
$$

We can then always set $b_{\infty} \rightarrow 0$. This puts the moduli matrix 2.8 in the form 2 ;

$$
b_{0}^{\prime}=\frac{\rho}{z-z_{0}},
$$

which describes a lump of position $z_{0}$ and the size $\rho$. By explicitly performing this rotation we obtain\},

$$
z_{0}=\frac{z_{S}+\left|b_{\infty}\right|^{2} z_{N}}{1+\left|b_{\infty}\right|^{2}}, \quad \rho=\frac{b_{\infty}}{1+\left|b_{\infty}\right|^{2}}\left(z_{S}-z_{N}\right)
$$

\section{Gauged $\mathbb{C} P^{1}$}

We now consider the gauged version of the $\mathbb{C} P^{1} \mathrm{NL} \sigma \mathrm{M}$ obtained from (2.1) by gauging the following $U(1)$ subgroup of the $S U(2)$ isometry (2.9)

$$
U(1):\left(\begin{array}{cc}
e^{-i \theta} & 0 \\
0 & e^{i \theta}
\end{array}\right), \quad u=e^{-i \theta}, \quad v=0
$$

which acts linearly on the field $b$

$$
b \rightarrow e^{-2 i \theta} b
$$

The lagrangian we obtain is then the following [35]:

$$
\mathcal{L}_{U(1)}=-\frac{1}{4 g^{2}} F_{g}^{\mu \nu} F_{g \mu \nu}+\xi \frac{\left|\nabla_{\mu} b\right|^{2}}{\left(1+|b|^{2}\right)^{2}}-\frac{g^{2}}{2}\left(\xi \frac{-2|b|^{2}}{1+|b|^{2}}-\zeta\right)^{2}
$$

where

$$
\nabla_{\mu}=\partial_{\mu}+2 i A_{g \mu}
$$

The complicated potential term is the necessary one for the existence of BPS saturated solitons. It can be more easily derived as a D-term potential which arises as one imposes supersymmetry 4 . In the language of supersymmetry, $\zeta$ is a Fayet-Iliopoulos term [36].

\footnotetext{
${ }^{2}$ If the expectation value of $b$ is vanishing, we must have a rational map where the degree of the numerator is less than the degree of the denominator.

${ }^{3}$ Intuitively, the center of the lump is mapped to $b\left(z_{0}\right)=-1$, the point diametrically opposed to $b_{\infty}=1$. The size is given by the displacement from the trivial map (zero size lump) $z_{S}=z_{N}=0$.

${ }^{4}$ See the Appendix for more details.
} 
The existence of BPS solitons requires that the potential term vanishes in the vacuum of the theory ${ }^{5}$. This occurs for

$$
\left|b_{\infty}\right|^{2}=-\frac{\zeta}{\zeta+2 \xi}
$$

The Fayet-Iliopoulos term must then assume values within the following range:

$$
-2 \xi \leq \zeta \leq 0
$$

In a generic vacuum the expectation value of $b$ spontaneously breaks the $U(1)$ gauge symmetry, and the model contains ANO (Abrikosov-Nielsen-Olesen) vortices [15] supported by the following homotopy group:

$$
\pi_{1}(U(1))=\mathbb{Z}
$$

Notice that all the discussions of this Section have been carried out considering only one coordinate patch for the $\mathbb{C} P^{1}$ target space. As already mentioned, to cover the full manifold we need to take into account another coordinate patch obtained with the change of variable:

$$
b^{\prime}=1 / b
$$

In the gauged case, after this change of variables the lagrangian (2.14) takes the form

$$
\mathcal{L}_{U(1)}=-\frac{1}{4 g^{2}} F_{g}^{\mu \nu} F_{g \mu \nu}+\xi \frac{\left|\nabla_{\mu} b^{\prime}\right|^{2}}{\left(1+\left|b^{\prime}\right|^{2}\right)^{2}}-\frac{g^{2}}{2}\left(\xi \frac{-2\left|b^{\prime}\right|^{2}}{1+\left|b^{\prime}\right|^{2}}-\zeta^{\prime}\right)^{2},
$$

which has the same form of (2.14) provided that the FI term $\zeta$ transforms nontrivially while we change coordinate patch:

$$
\zeta^{\prime}=-\zeta-2 \xi
$$

\section{$2.2 \quad$ Vortex equations}

The first step in the study of vortex solutions is to employ a Bogomol'nyi completion of the action (2.14) 30]:

$$
\begin{aligned}
\mathcal{E}_{U(1)} & =\frac{1}{2 g^{2}}\left[F_{g 12}-g^{2}\left(\xi \frac{-2|b|^{2}}{1+|b|^{2}}-\zeta\right)\right]^{2}+4 \xi \frac{\left|\nabla_{g \bar{z}} b\right|^{2}}{\left(1+|b|^{2}\right)^{2}}+ \\
& -\zeta F_{g 12}+\xi \epsilon_{i j} \partial_{i} \mathcal{N}_{j} . \\
\mathcal{N}_{j} & =\frac{i}{2\left(1+|b|^{2}\right)}\left(b \nabla_{j} \bar{b}-\bar{b} \nabla_{j} b\right) .
\end{aligned}
$$

\footnotetext{
${ }^{5}$ This is related to the existence of a supersymmetric vacuum in the supersymmetric extension of the model
} 
As usual, vortex equations are given by imposing the vanishing of the squares in the first line of the expression above:

$$
\begin{aligned}
F_{g 12} & =g^{2}\left(\xi \frac{-2|b|^{2}}{1+|b|^{2}}-\zeta\right) \\
\nabla_{g \bar{z}} b & =0 .
\end{aligned}
$$

The total tension is then given by the two surface terms in the second line. The first term is proportional to the magnetic flux density. The corresponding second term in linear sigma models is usually discarded as a vanishing boundary term. However, it is non-vanishing for compact NL $\sigma \mathrm{M}$. To give the correct topological interpretation to the two surface terms above, we have first to discuss in more details the topology supporting vortices in the $\mathbb{C} P^{1}$ NL $\sigma \mathrm{M}$. Vortices are undoubtedly characterized by the homotopy group (2.18). However, when we consider compact spaces, equation (2.18) does not give a complete classification of vortices [17]. We can in fact unwind the circle $b=\left|b_{\infty}\right| e^{i \theta}$ representing a vortex configuration at the boundary by either crossing the point $b=0$ ("south pole") or the point $b=\infty$ ("north pole"). The topology which correctly describes vortices including core structures in the gauged $\mathbb{C} P^{1}$ sigma model is then the following:

$$
\left.\pi_{1}(U(1))\right|_{\mathbb{C} P^{1}}=\mathbb{Z}_{S} \times \mathbb{Z}_{N}
$$

We will distinguish vortices with different $\mathbb{Z}$ charges by labeling them as $\mathrm{S}$ and $\mathrm{N}$ vortices. The corresponding magnetic fluxes (proportional to topological charges), as we shall prove soon, may then be written in the following way

$$
\begin{aligned}
\mathcal{V}_{j S} & =\mathcal{N}_{j} ; \\
\mathcal{V}_{j N} & =\mathcal{N}_{j}+2 A_{g j},
\end{aligned}
$$

and the energy density can be written as:

$$
\mathcal{E}_{U(1)}=\frac{\zeta+2 \xi}{2} \epsilon_{i j} \partial_{i} \mathcal{V}_{j S}-\frac{\zeta}{2} \epsilon_{i j} \partial_{i} \mathcal{V}_{j N}
$$

\section{Moduli matrix}

We now employ the moduli matrix approach [37,38 to construct vortices and their moduli space. The moduli matrix construction can be considered as a direct generalization of the rational map construction for lumps to the gauged case $[8,6,24]$. As usual, we do so by performing the following change of variables

$$
\begin{aligned}
b(z, \bar{z}) & =s^{2}(z, \bar{z}) b_{0}(z), \\
A_{g \bar{z}} & =-i \partial_{\bar{z}} \log s,
\end{aligned}
$$

where $s$ is a non-vanishing function, while $b_{0}$ is holomorphic. Notice that the change of variables above introduces an un-physical "V-equivalence" which scales $s$ and $b_{0}$ by multiplication with a constant $V$

$$
s^{2} \rightarrow V^{-1} s^{2}, \quad b_{0} \rightarrow V b_{0}
$$

\footnotetext{
${ }^{6}$ Similar connections between the moduli matrix and the rational map construction for monopoles were discussed in Ref. 39].
} 
but leaves all physical quantities unchanged. The second of the equations (2.23) is then identically solved, while the first reduces to a second order gauge invariant master equation:

$$
\partial_{\bar{z}} \partial_{z} \log \omega=-\frac{g^{2}}{4}\left(\xi \frac{-2 \omega^{2}\left|b_{0}\right|^{2}}{1+\omega^{2}\left|b_{0}\right|^{2}}-\zeta\right), \quad \omega=s s^{\dagger} .
$$

The holomorphic function $b(z)$ is called moduli matrix, and its complex coefficients parameterize the moduli space of vortices in the system considered. Let us assume that $b(z)$ has a finite number of zeroes and poles. Then it can be written as a ratio of polynomials

$$
b_{0}(z)=b_{\infty} \frac{z^{n_{S}}+p_{1}^{n_{S}-1}+\cdots+p_{n_{S}}}{z^{n_{N}}+q_{1}^{n_{N}-1}+\cdots+q_{n_{N}}} .
$$

In the expression above, the overall coefficient $b_{\infty}$ is not a modulus, since it can be fixed by $\mathrm{V}$-equivalence. We chose it to be equal to the expectation value of $b$. The master equation for $s$ must then be solved (numerically) with the following boundary conditions

$$
|s| \rightarrow|z|^{n_{N}-n_{S}} \quad \text { as } \quad|z| \rightarrow \infty .
$$

The coefficients $p_{i}$ and $q_{j}$ represent moduli of the vortex configuration, and the complex dimension of the moduli space is:

$$
\operatorname{Dim}_{\mathbb{C}} \mathcal{M}=n_{S}+n_{N} .
$$

Using the moduli matrix formalism we can now easily evaluate the flux densities (2.25)

$$
\begin{aligned}
& \int d x^{2}\left(\partial_{1} \mathcal{V}_{2 S}-\partial_{2} \mathcal{V}_{1 S}\right)=2 \int d x^{2} \partial_{z} \partial_{\bar{z}} \log \left(\frac{1+\omega^{2}\left|b_{0}\right|^{2}}{\omega^{2}\left|b_{0}\right|^{2}}\right)=2 \pi n_{S} \\
& \int d x^{2}\left(\partial_{1} \mathcal{V}_{2 N}-\partial_{2} \mathcal{V}_{1 N}\right)=2 \int d x^{2} \partial_{z} \partial_{\bar{z}} \log \left(1+\omega^{2}\left|b_{0}\right|^{2}\right)=2 \pi n_{N},
\end{aligned}
$$

which define the integers $n_{S}$ and $n_{N}$ appearing as the degree of the polynomials in moduli matrix as the $\mathrm{S}$ and N-vortex number:7 The total tension is then

$$
T_{U(1)}=\pi(\zeta+2 \xi) n_{S}-\pi \zeta n_{N} .
$$

Notice that the total magnetic flux is:

$$
2 \pi \nu_{v}=-\int d x^{2} F_{g 12}=-2 \int d x^{2} \partial_{z} \partial_{\bar{z}} \log \omega=\pi\left(n_{S}-n_{N}\right)
$$

thus $\mathrm{S}$ and $\mathrm{N}$-vortices have opposite $U(1)$ charges. We can also define a "fractional lump number"

$$
\nu_{l}=\frac{n_{S}+n_{N}}{2} .
$$

This definition is justified if we notice that the integer above is given, in fact, by the integral of following quantity:

$$
\left(\mathcal{V}_{j S}+\mathcal{V}_{j N}\right) / 2=\frac{i}{2\left(1+|b|^{2}\right)}\left(b \nabla_{j} \bar{b}-\bar{b} \nabla_{j} b\right)-A_{g j},
$$

which reduces to the lump number given in Eq. 2.4 for the un-gauged case when $A_{g j}$ vanishes.

\footnotetext{
${ }^{7}$ Since the function $s$ is non-vanishing, each zero (pole) of $b_{0}(z)$ clearly correspond to the only points where $b$ equals the value at the south or north pole, roughly corresponding to the cores of $\mathrm{S}$ and $\mathrm{N}$ vortices.
} 


\section{$2.3 \mathrm{~S}(\mathrm{~N})$ isolated vortex}

In this Section we construct a fundamental vortex and numerically solve the master equation (2.29). A fundamental S-vortex is given by the following choice for the moduli matrix:

$$
b_{0}(z)=b_{\infty}\left(z-z_{S}\right), \quad|\omega|^{2} \rightarrow|z|^{-1} .
$$

In fact, the vortex unwinds around the South pole $b=0$ at the center $z_{S}$ of the vortex. Notice from Eq. (2.36) that the S-vortex has fractional lump charge $\nu_{l}=1 / 2$. Similarly, we have an N-vortex with the choice:

$$
b_{0}(z)=b_{\infty} \frac{1}{z-z_{N}}, \quad|\omega|^{2} \rightarrow|z|
$$

where the vortex unwind around the point $b=\infty$ at the point $z_{N}$. The N-vortex also has fractional lump charge $\nu_{l}=1 / 2$.

Let us study a fundamental vortex in terms of $\zeta$. Fig. 3 shows the energy density profile of an S-vortex as a function of the FI term $\zeta$. The vortex disappears, by dilution, in the limit $\zeta \rightarrow 0$, where the gauge symmetry defined on the S-patch is unbroken. In the other limit $\zeta \rightarrow-2 \xi$, where the expectation value $b_{\infty}$ goes to infinity, the vortex becomes a spike. There is no need for additional work to study the N-vortex. From both the mass formula

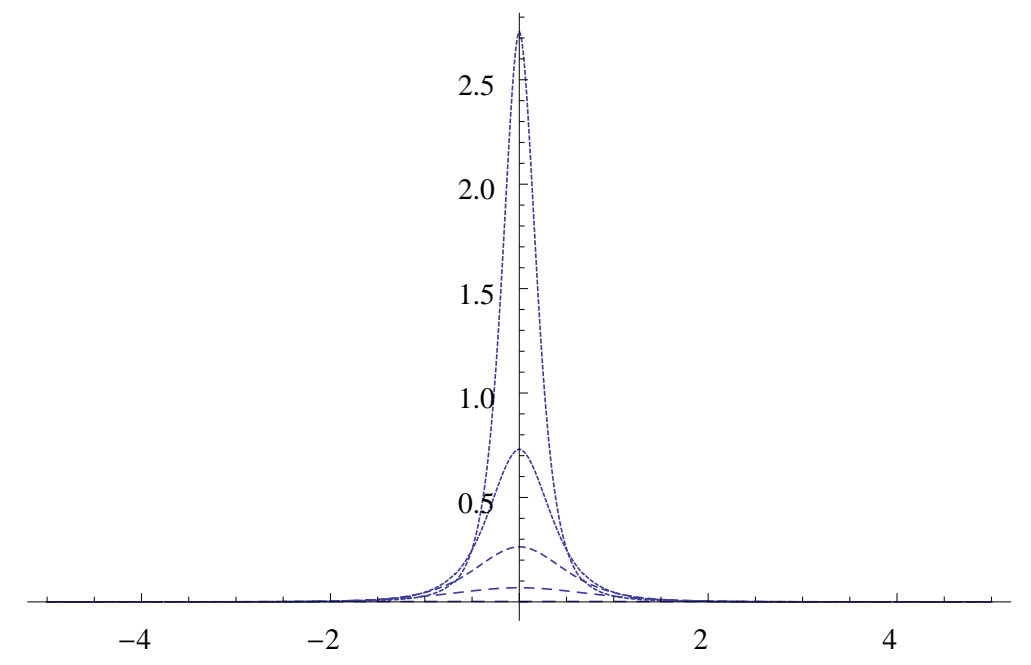

Figure 3: An S-vortex for various values of $\zeta . \xi=1, \zeta=-1.9,-1.45,-1,0.55,0.1, g=1$.

and the BPS equations, we see that an N-vortex is transformed into a physically equivalent S-vortex by the formal replacements:

$$
b \rightarrow 1 / b, \quad \zeta \rightarrow-\zeta-2 \xi .
$$

A choice of parameters which gives a narrow S-vortex will then gives a wide N-vortex and vice versa. This can also easily understood if we plot the potential of Eq. (2.14) as in Fig. 4 . If $\zeta$ is closer to $2 \xi$, the potential is higher around the S-pole than it is around the N-pole. A narrow $\mathrm{S}$ vortex is thus favored to minimize the potential energy with respect to the gradient 


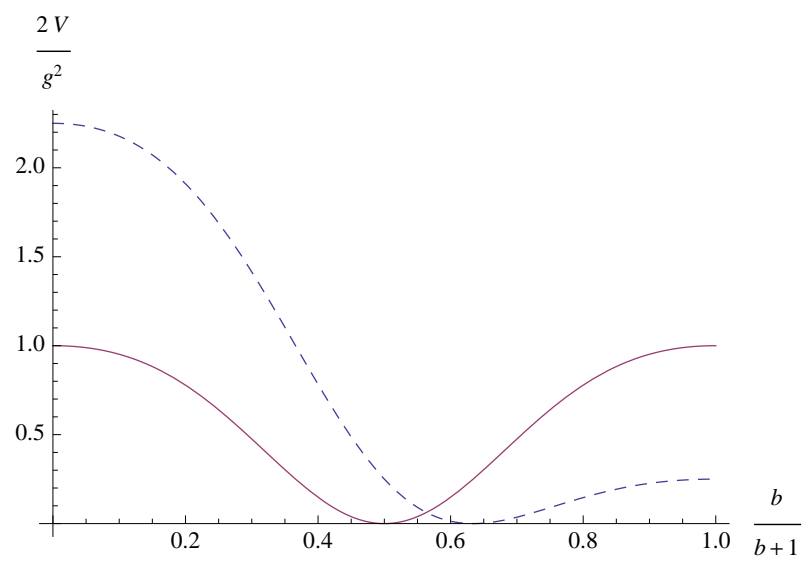

Figure 4: The potential as a function of $\beta=\frac{b}{1+b}$. This convenient combination takes into account the metric factor present in the kinetic term of $b$. Solid line: $\zeta=-1$. Dashed line: $\zeta=-1.5$.

energy (which tends to be higher for narrower configurations). Vice versa, for an N-vortex a wider configuration is favored. Notice that when $\zeta=-\xi$, the potential is symmetric and both vortices have the same width. We then have two independent effects which determine the size of vortices. A "core" effect, is related to the height of the potential which is different at the center of $\mathrm{S}$ and $\mathrm{N}$ vortices. A "tail" effect, instead, is related to the long distance, exponential fall-off of the vortex, and is the same for both $\mathrm{S}$ and $\mathrm{N}$ vortices. Analogously to the Abelian Higgs model case, we can estimate the typical sizes as follows

$$
\lambda_{S}=\frac{1}{g \xi\left|b_{\infty}\right|}, \quad \lambda_{N}=\frac{1}{g \xi\left|b_{\infty}^{\prime}\right|}=\frac{\left|b_{\infty}\right|}{g \xi}
$$

if we ignore the effects of the curvature of the target space on the vortex profile. The exponential tail can then be checked analytically expanding the master equation in terms of small fluctuations around the vacuum. We thus proceed expanding $\omega_{g}$ in the following way:

$$
\omega=\left|\frac{b_{\infty}}{b_{0}(z)}\right|\left(1+\delta \omega+\mathcal{O}\left(\delta \omega^{2}\right)\right) .
$$

The master equation $(2.29)$ then linearizes:

$$
\partial_{z} \partial_{\bar{z}} \delta \omega=g^{2} \xi \frac{\left|b_{\infty}\right|^{2}}{\left(1+\left|b_{\infty}\right|^{2}\right)^{2}} \delta \omega=-g^{2} \frac{\zeta(\zeta+2 \xi)}{4 \xi} \delta \omega \equiv \frac{\lambda}{4} \delta \omega
$$

The solution of the equation above is the well-known modified Bessel function of the second kind:

$$
\delta \omega(r)=K_{0}(\lambda r) \sim \frac{e^{-\lambda r}}{r}
$$

The exponential decay factor $\lambda$ corresponds to the mass of the $b$ field in the vacuum. Notice that $\lambda$ is invariant under change of coordinate, as it should be since it is a physical quantity. 


\section{S-N Vortex System}

If we send the gauge coupling to zero we have to recover the un-gauged sigma model, which does not admit vortices. In fact, the fundamental $\mathrm{S}$ or $\mathrm{N}$ vortices become wider in this limit, and eventually they vanish.

However, there is something interesting which happens when we take the limit $g \rightarrow 0$ in the presence of a composite configuration of $\mathrm{S}$ and $\mathrm{N}$ vortices: we may recover lump solutions of the un-gauged NL $\sigma \mathrm{M}$. We will study this phenomenon in this Section.

\subsection{Well separated S-N vortices}

Vortices are well separated when their typical widths are much smaller than their separations:

$$
\lambda_{S, N} \ll \Delta z_{i} .
$$

In this situation the moduli matrix 2.30 obviously represents a set of well separated $n_{N}$ $\mathrm{N}$-vortices located at the poles of $b_{0}$ and $n_{S}$ S-vortices located at zeroes. We also refer to this situation as "strong gauging", since Eq. (3.1) can be always satisfied with a sufficiently large value of the gauge coupling (see Eq. 2.41)).

The simplest configuration is given by the following choice:

$$
b_{0}(z)=b_{\infty} \frac{z-z_{S}}{z-z_{N}} .
$$

A numerical simulation in the case above is shown in Fig. 5 where an $\mathrm{S}$ and an $\mathrm{N}$ vortex are clearly identified when the gauge coupling is sufficiently large.

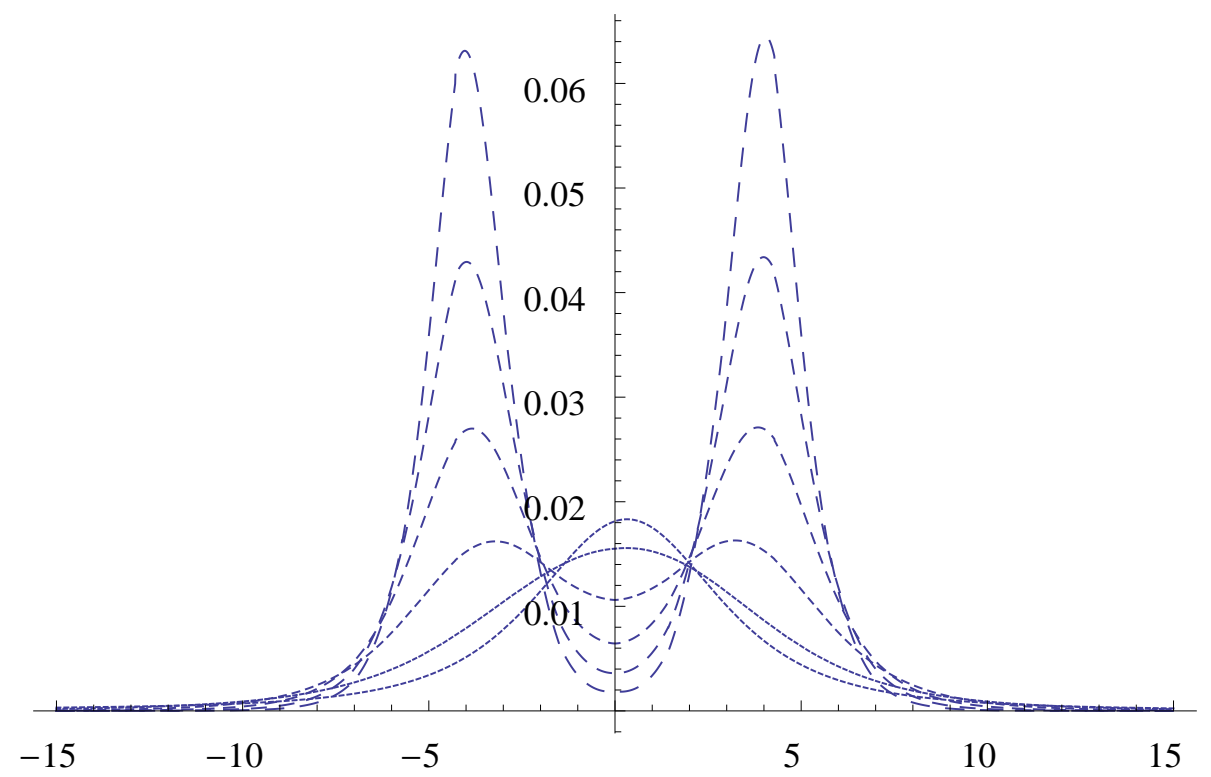

Figure 5: Deforming a lump into an N-S vortex system. $b_{\infty}=1, \xi=-\zeta=1, z_{S}=-z_{N}=-4$, $g=0,0.1,0.2,0.3,0.4,0.5$. 


\section{2 "Gauged" lumps}

Let us now consider the limit $g \rightarrow 0$. We want to recover the lump solutions in the original un-gauged NL $\sigma \mathrm{M}$ described by Eq. (2.7). Looking at the first line of Eq. (2.27) and the definition of the moduli matrix $b_{0}$ in Eq. 2.30) we see that $b$ must reduce to a purely holomorphic function. Assuming that all the holomorphic factors are extracted in the ratio $b_{0}(z)$, we have

$$
\omega \rightarrow 1 \text { as } g \rightarrow 0
$$

Moreover, if $b_{\infty} \neq 0$, the number of $\mathrm{N}$ and $\mathrm{S}$ vortices must be the same:

$$
n_{S}=n_{N}=N \text {. }
$$

In this situation the lump number defined in Eq. (2.4) is a well-defined integer. If the gauge coupling is small enough (weak gauging regime), we expect to find solitonic solutions similar to lumps of the un-gauged model at small distances. Nevertheless, at large distances this solitons will show their true nature of vortices falling off with an exponential factor. This behavior can be confirmed with numerical simulations. Again, Fig. 5 shows how a lump, in the weak gauging regime, which is deformed into a composite state of $\mathrm{S}$ and $\mathrm{N}$ vortices once the gauge coupling is strong enough.

We can also perform, to some extent, an analytical analysis of the weak coupling limit, by expanding the non-holomorphic term $w$ and the master equation (2.29) as follows:

$$
\begin{aligned}
w & =1+\delta w+\mathcal{O}\left(g^{2}\right), \\
\partial_{\bar{z}} \partial_{z} \delta \omega & =-\frac{g^{2}}{4}\left(\xi \frac{-2\left|b_{0}\right|^{2}}{1+\left|b_{0}\right|^{2}}-\zeta\right)+\mathcal{O}\left(g^{2}\right) .
\end{aligned}
$$

Let us study the equation above in the proximity of a zero (pole) around $z=z_{0}$. The second term can be approximated by a constant in the vicinity of $z_{0}$ :

$$
\partial_{\bar{z}} \partial_{z} \delta \omega=\frac{g^{2}}{4}(\zeta+\xi \pm \xi), \quad\left|z-z_{0}\right| \ll \Delta z_{i},
$$

where the $\Delta z_{i}$ are the typical separations between zeros and poles. The plus and minus signs respectively apply to the case of zeroes and poles. The equation above can then be easily solved for $\delta \omega$ :

$$
\delta \omega \sim \frac{g^{2}}{4}(\zeta+\xi \pm \xi)\left|z-z_{0}\right|^{2}+\text { harmonics }
$$

where with harmonics we mean solution of the homogenous equation $\partial_{\bar{z}} \partial_{z} \delta \omega=0^{8}$. Since the non-homogeneous term grows with the distance from $z_{0}$, the lump approximation is valid when:

$$
\left|z-z_{0}\right|^{2} \ll\left|\Delta z_{i}\right|^{2} \ll\left(\frac{g^{2}}{4}(\zeta+\xi \pm \xi)\right)^{-1} .
$$

\footnotetext{
${ }^{8}$ These are fluctuating solutions which are not important in the present argument.
} 
The condition above also tells us that a generic configuration of $\mathrm{S}$ and $\mathrm{N}$ vortices is approximated by a lump solution whenever the typical distances $\left|\Delta z_{i}\right|$ are small enough. Notice also that the approximation above is valid at short distances only. At sufficiently large distances, in fact, we have to spot the true "local" nature of the configuration for finite $g$. Indeed, the second term of Eq. (3.5) vanishes at large distances, and we have to take into account terms linear in $\delta \omega$ in the expansion. We then recover Eq. (2.42) which gives the correct exponential tail.

The metric on the moduli space of composite $\mathrm{S}$ and $\mathrm{N}$ vortices was also previously studied in 17,18 , where it was found that a singularity develops when a couple of $\mathrm{S}-\mathrm{N}$ vortices coincides. In this Section we have shown that a configuration of two very close vortices (a pole and a zero) is indistinguishable from a lump (see Eq. (2.11). The singularity discovered in Refs. 17, 18 is then exactly identified as a small lump singularity. As well-known, small lump singularities can be "resolved" by introducing an appropriate UV completion for the NL $\sigma \mathrm{M}$. In the next Section we will consider this possibility explicitly.

\section{Linear Formulation}

\subsection{Model}

It is simple to guess the correct UV completion of the gauged $\mathbb{C} P^{1} \mathrm{NL} \sigma \mathrm{M}$. First let us consider the un-gauged NL $\sigma \mathrm{M}$. It can be easily written as the strong gauge limit $e \rightarrow \infty$ of the so-called extended Abelian Higgs model with two flavors:

$$
\mathcal{L}=-\frac{1}{4 e^{2}} F_{e}^{\mu \nu} F_{e \mu \nu}+\left|\nabla_{\mu} \phi_{1}\right|^{2}+\left|\nabla_{\mu} \phi_{2}\right|^{2}-\frac{e^{2}}{2}\left(\left|\phi_{1}\right|^{2}+\left|\phi_{2}\right|^{2}-\xi\right)^{2} .
$$

As well-known, the model above admits semi-local vortices which are a particular type of strings which admit size moduli [6 10,24]. They are indeed very similar to lump solutions of the underlying $\mathbb{C} P^{1} \mathrm{NL} \sigma \mathrm{M}$ (to which they reduce in the $e \rightarrow \infty$ limit). However, while a lump becomes a singular spike in the zero size limit, a semi-local vortex remains regular and reduces to a traditional (sometimes called "local") Abrikosov-Nielesen-Olsen vortex, which has a typical size of order $1 / e \sqrt{\xi}$. In this sense, the moduli space of semi-local vortices is considered to be a regularization of the singularities (small lump singularities) of the lump moduli space.

Semi-local vortices ultimately exist because the vacuum manifold, in this case $S^{3}$, is simply connected

$$
\pi_{1}\left(S^{3}\right)=0
$$

while the second homotopy group of the moduli space of vacua $S^{3} / U(1)=\mathbb{C} P^{1}$ is non trivial [10]

$$
\pi_{2}\left(\mathbb{C} P^{1}\right)=\mathbb{Z}
$$

Notice that the $S U(2)$ isometry of the $\mathbb{C} P^{1} \mathrm{NL} \sigma \mathrm{M}$ is now seen as a flavor symmetry which rotates the fields $\phi_{i}$. Moreover, we can identify the holomorphic coordinate $b$ appearing in 
Eq. (2.1) as:

$$
b=\frac{\phi_{2}}{\phi_{1}} .
$$

It is then clear that to obtain the model 2.14 in the $e \rightarrow \infty$ we have to gauge a $U(1)$ flavor symmetry with the charge assignments given in Table 1 . The resulting model is then the

\begin{tabular}{l|cc}
$U(1)^{2}$ & $\phi_{1}$ & $\phi_{2}$ \\
\hline$U(1)_{e}$ & 1 & 1 \\
$U(1)_{g}$ & 1 & -1
\end{tabular}

Table 1: Field content and charges

following linear gauged model

$$
\begin{aligned}
\mathcal{L} & =-\frac{1}{4 e^{2}} F_{e}^{\mu \nu} F_{e \mu \nu}-\frac{1}{4 g^{2}} F_{g}^{\mu \nu} F_{g \mu \nu}+\left|\nabla_{\mu} \phi_{1}\right|^{2}+\left|\nabla_{\mu} \phi_{2}\right|^{2}+ \\
& -\frac{e^{2}}{2}\left(\left|\phi_{1}\right|^{2}+\left|\phi_{2}\right|^{2}-\xi\right)^{2}-\frac{g^{2}}{2}\left(\left|\phi_{1}\right|^{2}-\left|\phi_{2}\right|^{2}-\chi\right)^{2},
\end{aligned}
$$

where $\chi$ is a second FI term for the new gauge group $U(1)_{g}$ and

$$
\nabla_{\mu} \phi_{1}=\left(\partial_{\mu}-i A_{e \mu}-i A_{g \mu}\right) \phi_{1}, \quad \nabla_{\mu} \phi_{2}=\left(\partial_{\mu}-i A_{e \mu}+i A_{g \mu}\right) \phi_{2} .
$$

By comparing the second potential term in the equation above with the potential term arising in the gauged NL $\sigma \mathrm{M}$ lagrangian (2.14) we also obtain the following relationship between the FI terms $\chi$ and $\zeta$

$$
\chi=\zeta+\xi
$$

The vacuum of the theory is then given by:

$$
\left|\phi_{1 \infty}\right|^{2}=\frac{\xi+\chi}{2}, \quad\left|\phi_{2 \infty}\right|^{2}=\frac{\xi-\chi}{2}
$$

Both the $U(1)$ gauge groups are then spontaneously broken, we thus have for the first homotopy group:

$$
\pi_{1}\left(\frac{U(1)_{e} \times U(1)_{g}}{\mathbb{Z}_{2}}\right)=\mathbb{Z}_{S} \times \mathbb{Z}_{N}
$$

Notice that, due to the presence of a $\mathbb{Z}_{2}$ common factor, the smallest closed paths are obtained with a $\pi$ rotation into the group $U(1)_{e}$ and either a $\pi$ or a $-\pi$ rotation into the group $U(1)_{g}$. These two paths represent the smallest elements of $\mathbb{Z}_{S}$ and $\mathbb{Z}_{N}$ respectively. The choice of notation is not a coincidence either: the two factors characterize two type of vortices which correspond to the $\mathrm{S}$ and $\mathrm{N}$ vortices we already studied in the previous Sections in the $e \rightarrow \infty$ limit. The $\mathrm{S}$ and $\mathrm{N}$ vortices are a particular type of what is called, in 
literature, fractional vortex. They were first discovered in two component superconductors within a Landau-Ginzburg model [27], which can be considered as a non-relativistic version of the model (4.5) where, in the case of superconductors, the group $U(1)_{g}$ is global ${ }^{9}$. This definition is due to the fact that the fundamental vortices carry only one half of the magnetic flux of both gauge groups (in particular of the group $\left.U(1)_{e}\right)$. For generic values of the gauge couplings, the $\mathrm{S}$ and $\mathrm{N}$ vortices do not feel static interactions, even if they form a coupled system. Non-static interactions are on the other hand non-trivial and can be studied in the moduli space approximation [40]. However, when the gauge couplings coincide $e=g$ the S and $\mathrm{N}$ vortices completely decouple and do not interact at all, at least at the classical.

\subsection{Vortices: BPS equations and moduli matices}

As usual we can perform a Bogomol'nyi completion of the action

$$
\begin{aligned}
\mathcal{E} & =\frac{1}{2 e^{2}}\left[F_{e 12}-e^{2}\left(\left|\phi_{1}\right|^{2}+\left|\phi_{2}\right|^{2}-\xi\right)\right]^{2}+\frac{1}{2 g^{2}}\left[F_{g 12}-g^{2}\left(\left|\phi_{1}\right|^{2}-\left|\phi_{2}\right|^{2}-\chi\right)\right]^{2}+ \\
& +4\left|\nabla_{\bar{z}} \phi_{1}\right|^{2}+4\left|\nabla_{\bar{z}} \phi_{2}\right|^{2}-\xi F_{e 12}-\chi F_{g 12}+2 \partial_{z} \partial_{\bar{z}}\left(\left|\phi_{1}\right|^{2}+\left|\phi_{2}\right|^{2}\right),
\end{aligned}
$$

which leads to BPS equations [16]:

$$
\begin{aligned}
F_{e 12} & =e^{2}\left(\left|\phi_{1}\right|^{2}+\left|\phi_{2}\right|^{2}-\xi\right) \\
F_{g 12} & =g^{2}\left(\left|\phi_{1}\right|^{2}-\left|\phi_{2}\right|^{2}-\chi\right) \\
\nabla_{\bar{z}} \phi_{1} & =\nabla_{\bar{z}} \phi_{2}=0 .
\end{aligned}
$$

The tension is then given by the following topological term:

$$
T=2 \pi \xi \nu_{e}+2 \pi \chi \nu_{g}
$$

where $\nu_{e}$ and $\nu_{g}$ are respectively the winding numbers for the gauge groups $U(1)_{e}$ and $U(1)_{g}$. Notice that the last term in Eq. 4.10) is a total derivative with vanishing contribution.

The moduli matrix formalism is implemented as usual with the substitutions [41]:

$$
\begin{aligned}
\phi_{1}(z, \bar{z}) & =s_{e}^{-1}(z, \bar{z}) s_{g}^{-1}(z, \bar{z}) \phi_{01}(z), \\
\phi_{2}(z, \bar{z}) & =s_{e}^{-1}(z, \bar{z}) s_{g}(z, \bar{z}) \phi_{02}(z), \\
A_{e \bar{z}} & =-i \partial_{\bar{z}} \log s_{e}, \\
A_{g \bar{z}} & =-i \partial_{\bar{z}} \log s_{g}, \\
\omega_{e}=\left|s_{e}\right|^{2}, & \omega_{g}=\left|s_{g}\right|^{2} .
\end{aligned}
$$

$\phi_{01}(z)$ and $\phi_{02}(z)$ are the moduli matrices for $\phi_{1}$ and $\phi_{2}$ respectively. The general prescription to find the right boundary conditions on the moduli matrix was described in Ref. [41] and gives in the present case

$$
\begin{aligned}
\phi_{01}(z) & =\sqrt{\frac{\xi+\chi}{2}}\left(z^{n_{N}}+p_{1} z^{n_{N}-1}+\cdots+p_{n_{N}}\right) \\
\phi_{02}(z) & =\sqrt{\frac{\xi-\chi}{2}}\left(z^{n_{S}}+q_{1} z^{n_{S}-1}+\cdots+q_{n_{S}}\right) \\
\omega_{e} \rightarrow|z|^{n_{N}+n_{S}}, & \omega_{g} \rightarrow|z|^{n_{N}-n_{S}}
\end{aligned}
$$

\footnotetext{
${ }^{9}$ Different types of fractional vortices were also introduced in Ref. 12,13 .
} 
where $n_{S}$ and $n_{N}$ are the vortex numbers

$$
\begin{aligned}
& \nu_{e}=-\frac{1}{2 \pi} \int d x^{2} F_{e 12}=-\frac{1}{\pi} \int d x^{2} \partial_{z} \partial_{\bar{z}} \log \omega_{e}=\frac{n_{S}+n_{N}}{2}, \\
& \nu_{g}=-\frac{1}{2 \pi} \int d x^{2} F_{g 12}=-\frac{1}{\pi} \int d x^{2} \partial_{z} \partial_{\bar{z}} \log \omega_{g}=\frac{n_{S}-n_{N}}{2} .
\end{aligned}
$$

In the language of the $\mathrm{NL} \sigma \mathrm{M}$ the integers above correspond to the (fractional) vortex and lump numbers defined in the previous section:

$$
\begin{aligned}
& \nu_{g}=\nu_{v} \\
& \nu_{e}=\nu_{l} .
\end{aligned}
$$

Finally, let us write the BPS equations in terms of the moduli matrices:

$$
\begin{aligned}
\partial_{\bar{z}} \partial_{z} \log \omega_{e} & =\frac{e^{2}}{4}\left(\omega_{e}^{-1} \omega_{g}^{-1}\left|\phi_{01}\right|^{2}+\omega_{e}^{-1} \omega_{g}\left|\phi_{02}\right|^{2}-\xi\right) \\
\partial_{\bar{z}} \partial_{z} \log \omega_{g} & =\frac{g^{2}}{4}\left(\omega_{e}^{-1} \omega_{g}^{-1}\left|\phi_{01}\right|^{2}-\omega_{e}^{-1} \omega_{g}\left|\phi_{02}\right|^{2}-\chi\right) .
\end{aligned}
$$

\subsection{Resolving the small lump singularity}

One can easily check that all the analysis of this Section correctly reduces to that done for the gauged $\mathbb{C} P^{1} \mathrm{NL} \sigma \mathrm{M}$ in the limit $e \rightarrow \infty$. Moreover, at finite $e$, but in the regime $e \gg g$, the results of the sections about the gauged NL $\sigma \mathrm{M}$ qualitatively apply to the linear case. We have just to substitute lumps with the very similar semi-local vortices in the discussions of the previous Sections. A semi-local vortex is thus split into a couple of $\mathrm{S}$ and $\mathrm{N}$ vortices upon gauging of a $U(1)$ flavor symmetry.

However, the crucial difference is that small lump singularities are now removed. As we have seen in the previous section, these singularities arise when a couple of $\mathrm{S}$ and $\mathrm{N}$ vortices have coincident positions: there, a singular spiky lump develops. At finite $e$, however, this singularity is substituted by the insertion of a regular local vortex. To see this, let us consider the moduli matrices in the case of two coincident $\mathrm{S}$ and $\mathrm{N}$ vortices. For simplicity, we can set $\chi=0$. Then we have

$$
\phi_{01}=\phi_{02}=\frac{\xi}{2}\left(z-z_{0}\right)
$$

and the BPS equations 4.17) can be reduced to the following:

$$
\begin{aligned}
\omega_{g} & \equiv 1 \\
\partial_{\bar{z}} \partial_{z} \log \omega_{e} & =-\frac{e^{2}}{4}\left(2 \omega_{e}^{-1}\left|\phi_{01}\right|^{2}-\xi\right)=-\frac{e^{2}}{2}\left(\omega_{e}^{-1}\left|\phi_{01}\right|^{2}-\xi / 2\right),
\end{aligned}
$$

which represent the master equation for a standard, regular vortex for the $U(1)_{e}$ group. 


\section{Regularization of the Semi-Local Vortex Metric}

As well-known, the effective theory on the world-sheet of semi-local vortices (and similarly of lumps) has an infrared logarithmic divergence due to the slow polynomial decay of the fields [8,6, 24,23]. As usual for effective theories with at least four supercharges, the effective theory can be conveniently written in terms of a Kähler potential. In the case of a semi-local vortex the Kähler potential can be computed exactly at each order in a power expansion in terms of $1 /(e|\rho| \sqrt{\xi})$, where $|\rho|$ is the size modulus of the semi-local vortex [42]. At the leading order in this expansion, the Kähler potential for a semi-local vortex in the Abelian extended Higgs model 4.1, is the following 10

$$
K\left(\left|z-z_{0}\right|^{2},|\rho|^{2}\right)=2 \pi \xi\left|z-z_{0}\right|^{2}+\pi \xi|\rho|^{2} \ln \left(\frac{L^{2}}{|\rho|^{2}}\right)+\pi \xi|\rho|^{2},
$$

where we included the first term which describes the position $z_{0}$ of the vortex. In the expression above, the divergent logarithm has been regularized with the introduction of a large infrared cut-off $L$.

So far, various different approaches have been considered to consistently deal with these divergences. Two possibilities were discussed in Ref. [23]. One is to consider strings of finite length $L$. This would naturally cut-off the divergences as in (5.1). However, this approach obviously spoils the BPS nature of the string. A more convenient way to proceed is to introduce a twisted mass $m$ for the size modulus $\rho$. The logarithm is then cut off at the scale $L \sim 1 / m$. The clear advantage of this approach is that the massive deformation preserve the BPS nature of the vortex. However, as a drawback, this regularization is ultimately due to the fact that we really lift the moduli space once we give a mass to the size parameter $\rho$. Another recent proposal takes advantage of the presence of the divergencies and of the possibility to eliminate them with an appropriate change of variables [42]. The effective action is then exactly given by just this rescaled divergent terms.

In this work, we propose a new rather interesting regularization of the metric on the moduli space of semi-local vortices which preserves the BPS nature of the string and does not lift its moduli space. As a consequence $\rho$ remains a genuinely massless zero mode. This regularization is obtained by weakly gauging a flavor symmetry. While the regularization through a massive deformation corresponds to the inclusion F-terms, or potential terms, into the effective action, the proposed regularization through weak gauging corresponds to a deformation of the Kähler potential. The example we studied in the previous Section is the explicit realization of this idea for the semi-local vortex in the extended Abelian Higgs model with two flavors, when we can gauge the relative phase between the two fields.

Recalling the discussion of the previous Sections, it is straightforward to determine how the divergent Kähler potential in (5.1) gets deformed, at least in two special limits. The first one is when the size of the vortex is small $|\rho|^{2} \ll 1 / g^{2} \xi$ :

$$
K\left(\left|z-z_{0}\right|^{2},|\rho|^{2}\right)=2 \pi \xi\left|z-z_{0}\right|^{2}+\pi \xi|\rho|^{2} \ln \left(\frac{1}{|\rho|^{2} g^{2} \xi}\right)+\pi \xi|\rho|^{2} .
$$

The expression above can be easily guessed if we notice that after gauging the power law fall-off of the fields is cut off with an exponential behavior, at distances of order of the mass

\footnotetext{
${ }^{10}$ High order corrections have been calculated in 42 .
} 
of the lightest particles in the bulk. In the limit of large size $|\rho|^{2} g^{2} \xi \gg 1$, on the other hand, the semi-local vortex is split into two local vortices, and the Kähler potential reduces to that of two isolated Abelian vortices 11 .

$$
K\left(\left|z-z_{N}\right|^{2},\left|z-z_{S}\right|^{2}\right)=\pi \xi\left|z-z_{N}\right|^{2}+\pi \xi\left|z-z_{S}\right|^{2}=2 \pi \xi\left|z-z_{0}\right|^{2}+2 \pi \xi|\rho|^{2},
$$

The Kähler potential at generic values of the coupling $g$ must then be calculated numerically using the expression

$$
\begin{aligned}
K\left(\left|z-z_{0}\right|^{2},|\rho|^{2}\right) & =\int d^{2} x\left\{\xi \ln \omega_{e}+\chi \ln \omega_{g}+\frac{1}{e^{2}} \partial_{i} \ln \omega_{e} \partial_{i} \ln \omega_{e}+\frac{1}{g^{2}} \partial_{i} \ln \omega_{g} \partial_{i} \ln \omega_{g}+\right. \\
& \left.+\omega_{e}^{-1} \omega_{g}^{-1}\left|\phi_{01}\right|^{2}+\omega_{e}^{-1} \omega_{g}\left|\phi_{02}\right|^{2}\right\}
\end{aligned}
$$

whose numerical evaluation must interpolates between the expressions $(5.2)$ and $(5.3)$.

\section{Generalizations}

In the case of the $\mathbb{C} P^{1} \mathrm{NL} \sigma \mathrm{M}$, the $U(1)$ sub-group of the $S U(2)$ isometry is the maximal symmetry which can be gauged without breaking supersymmetry [35]. This fact can be intuitively understood by recalling that gauging in supersymmetric theories generically reduces the complex dimension $D$ of a target manifold to $D-d$, where $d$ is the dimension of the group being gauged ${ }^{12}$. At most, we can then gauge $D$ isometries.

The discussion of the previous sections can be generalized in the case of NL $\sigma \mathrm{Ms}$ with higher dimensional target spaces $D>1$ in a variety of ways. Gauging in a different way the isometries of the target space will reveal different interesting connections between various type of vortices an lumps. In this Section we will have a brief look at some interesting possibilities.

\section{$6.1 \mathbb{C} P^{N-1} \mathrm{NL} \sigma \mathrm{M}$}

The $\mathbb{C} P^{N-1} \mathrm{NL} \sigma \mathrm{M}$ has a $S U(N)$ isometry. As already explained, gauging of the full isometry will break supersymmetry [35]. We can however safely consider the gauging of a subgroup of dimension at most $N-1$. We will consider, as particular examples, gauging of Abelian and non-Abelian subgroups.

\section{Gauging of an Abelian isometry}

A simple generalization of the model discussed in Section 2 is the one given by weakly gauging a $U(1)^{d}$ subgroup with charge assignments given by Table 2 . After gauging, the target space of $\mathbb{C} P^{N-1}$ is effectively reduced to $\mathbb{C} P^{N-1-d}$. When gauge couplings are sent to infinity,

\footnotetext{
${ }^{11}$ We have set $\chi=0$ for simplicity, throughout this Section.

${ }^{12}$ Gauging in a supersymmetric compatible way requires the introduction of a D-term potential, whose minimization gives generically $d$ real constraints, in addition to the $d$ degrees of freedom eaten by gauge invariance.
} 


\begin{tabular}{c|ccccc}
$U(1)^{d}$ & $b_{1}$ & $\ldots$ & $b_{d}$ & $\ldots$ & $b_{N-1}$ \\
\hline$U(1)_{1}$ & -2 & 0 & 0 & 0 & 0 \\
$\vdots$ & 0 & $\ddots$ & 0 & 0 & 0 \\
$U(1)_{d}$ & 0 & 0 & -2 & 0 & 0
\end{tabular}

Table 2: Field content and charges

then, the model supports $\mathbb{C} P^{N-1-d}$ lumps. Moreover, at finite values of gauge couplings, the Abelian gauge symmetries are generically spontaneously broken, and the model will support abelian vortices too:

$$
\pi_{1}\left(U(1)^{d}\right)=\mathbb{Z}^{d}
$$

Much in the same way of the $\mathbb{C} P^{1}$ case, a lump of the un-gauged $\mathbb{C} P^{N-1}$ sigma model will thus split into a composite state of a semi-local vortex (which reduces to a $\mathbb{C} P^{N-1-d}$ lump in the infinite gauge coupling limit) plus $d$ Abelian vortices. In other words, $d$ size moduli of the original lump are transformed into an equivalent number of position moduli of Abelian vortices.

\section{Gauging of a non-Abelian isometry}

Another interesting possibility is to gauge a non-Abelian $U(d)$ isometry, with $d^{2} \leq N-1$. As shown in Table 3, we can arrange $d^{2}$ fields as $d$ fundamentals of $U(d)$, while the rest are singlets. Again, after gauging the target space effectively reduces to $\mathbb{C} P^{N-1-d^{2}}$, which still

\begin{tabular}{c|c|c} 
& $b_{1}, \ldots, b_{d^{2}}$ & $b_{d^{2}+1}, \ldots, b_{N-1}$ \\
\hline$U(d)$ & $\square$ & 0
\end{tabular}

Table 3: Field content and charges

supports lumps. Moreover, non-Abelian vortices are supported by the non-trivial homotopy:

$$
\pi_{1}(U(d))=\mathbb{Z}
$$

After gauging of a $U(d)$ isometry then, lumps of $\mathbb{C} P^{N-1}$ sigma models reduce to a composite state of a semi-local vortex (similar to a $\mathbb{C} P^{N-1-d^{2}}$ lump) plus $d U(d)$ non-Abelian vortices. The presence of $d$ vortices is due to the fact that non-Abelian vortices have $1 / d$ charge with respect to an Abelian vortex. It can also be guessed by matching the dimensions of the moduli spaces. $d^{2}$ size moduli of the original lump are translated into $d$ orientations of the $d$ non-Abelian vortices.

\subsection{Grassmannian NL $\sigma \mathrm{M}$}

Grassmannian $\left(G r_{N, \tilde{N}}\right)$ can be considered as a generalization of $\mathbb{C} P^{N-1}$ manifolds. $G r_{N, \tilde{N}}$ is the set of $N$-dimensional complex planes in a $N+\tilde{N}$ dimensional space. They can be 
algebraically defined as the set of $N \times(\tilde{N}+N)$ matrices modulo an $S U(N)$ equivalence:

$$
G r_{N, \tilde{N}}=\left\{M_{N, \tilde{N}+N} \mid M \sim G M, G \in G L(\mathbb{C}, N)\right\}
$$

The complex dimension of the manifold is then $N \tilde{N}$. Grassmannian manifolds enjoy a $S U(N+\tilde{N})$ isometry. If we write the matrix $M$ as follows:

$$
M=(A \mid B)
$$

the isometry action is then:

$$
M \rightarrow M G_{S U(N+\tilde{N})}
$$

The matrices $A$ and $B$ have the role of homogeneous coordinates. Using the $G L(N, \mathbb{C})$ action, and assuming that $A$ is invertible, we can always reduce $M$ to the following form:

$$
M \sim\left(\mathbf{1} \mid B^{\prime}\right),
$$

where now the $B^{\prime}$ fields are the independent $N \tilde{N}$ holomorphic coordinates of the grassmannian ${ }^{13}$. Grassmannian lumps where studied in connection to non-Abelian semi-local vortices in Ref. [24, 44]. The dimension of the moduli space of a grassmannian lump is $N+\tilde{N}$.

Assuming $\tilde{N} \leq N$, we can choose to gauge a $U(\tilde{N})$ subgroup of the $S U(N \times \tilde{N})$ isometry acting on the fields like

$$
A \rightarrow A e^{-i \theta}, \quad B \rightarrow B G_{S U(\tilde{N})} e^{i \theta} .
$$

The original $G r_{N, \tilde{N}}$ manifold is then reduced to a gauged $G r_{\tilde{N}, N-\tilde{N}}$ manifold. The gauged sigma model will then support non-Abelian $U(\tilde{N})$ semi-local vortices, whose moduli space dimension is $\tilde{N}+(N-\tilde{N})=N$. However, the original grassmannian lump will split into a composite state of a $U(\tilde{N})$ semi-local vortex (which reduces to a $G r_{\tilde{N}, N-\tilde{N}}$ lump in the infinite gauge coupling limit) plus an additional $U(\tilde{N})$ local vortex.

\section{Summary and Conclusions}

In this paper we have explicitly constructed vortices in the $U(1)$ gauged $\mathbb{C} P^{1}$ non-linear sigma model, and studied them numerically. We identified the configuration given by the superposition of $\mathrm{N}$ and $\mathrm{S}$ vortices as a two-dimensional instanton (lump) of the original ungauged sigma model. In this way we identified a general mechanism to decompose instantons into smaller constituents by gauging an isometry of the target space of a non-linear sigma model.

We can perform a similar analysis in the linear case of the Abelian extended Higgs model with two flavors where a $U(1)$ flavor symmetry is also gauged. This model can be seen as an UV completion of the gauged $\mathbb{C} P^{1}$ sigma model. The extended Higgs model is wellknown to contain semi-local vortices [6,7]. In this context we identified the gauging of flavor

\footnotetext{
${ }^{13}$ Grassmannian manifolds are interesting in quantum field theory since they give the Higgs branch of supersymmetric $U(N) \mathcal{N}=2$ QCD with $N+\tilde{N}$ fundamental flavors 43 .
} 
symmetries as a general mechanism to regularize the metric of semi-local vortices. Contrarily to other known methods, the one we propose does not lift any moduli space parameter.

We also briefly discussed how the above ideas can be generalized to the case of nonlinear sigma models with higher dimensional target spaces. In general, lumps are split into component objects which can be identified as being vortices, non-Abelian vortices or semilocal vortices, depending on the chosen isometry one decides to gauge.

It is tantalizing to try to extend these ideas to the four dimensional case. An interesting direct connection between two dimensional lumps and four dimensional instantons has been found in Ref. [45] through non-Abelian vortices [38, 46, 22, 21, 47]. Precisely, instantons living in the un-broken phase of four dimensional non-Abelian $S U(N)$ gauge theories are confined on the two dimensional string world-sheet of non-Abelian vortices once one enters a broken Higgs phase. The effective theory of non-Abelian vortices is described by a two dimensional $\mathbb{C} P^{N-1}$ non-linear sigma model and instantons are then confined as lumps of the effective theory. Due to the results of the present paper, it is natural to expect that gauging of $U(1)$ flavor symmetries in the four dimensional theory may lead to instantons splitting into meron like objects. A situation where this set-up can be realized in nature is for example the color-flavor-locked superconducting phase in high density QCD [48], where non-Abelian vortices [49] admit $\mathbb{C} P^{2}$ moduli in the world-sheets [50] and the role of the additional $U(1)$ gauge symmetry is played by the electromagnetic interactions. Fractional instantons, or merons, on the world-sheet of non-Abelian strings have already been considered in Ref. [51] in the different context of $\mathcal{N}=1^{*} S U(N)$ gauge theories, where non-Abelian vortices are described by a non-supersymmetric, un-gauged $O(3)$ sigma model [52]. In this case merons are identified as global vortices instead of our local vortices. Moreover, the setup is completely non supersymmetric. We strongly believe that future research along this line may lead to new crucial understandings of the vacuum structure of strongly coupled gauge theories.

\section{Acknowledgments}

The work of MN is supported in part by Grant-in Aid for Scientific Research (No. 23740198) and by the "Topological Quantum Phenomena" Grant-in Aid for Scientific Research on Innovative Areas (No. 23103515) from the Ministry of Education, Culture, Sports, Science and Technology (MEXT) of Japan.

The work of WV is supported by the DOE grant DE-FG02-94ER40823. 


\section{A Superfield Formalism}

Let us explicitly derive the Bogomol'nyi equations for a general $U(1)$ gauged $\mathcal{N}=2$ nonlinear sigma-model. Using an $\mathcal{N}=1$ superfield formalism, and restricting to the relevant bosonic content, the action of the model is given in terms of a Kähler potential and a gauge kinetic term 35 :

$$
\begin{aligned}
\mathcal{L}_{K} & =\int d^{4} \theta\left\{K\left(e^{-V}\left|\Phi_{I}\right|^{2}\right)+\zeta V\right\}+\frac{1}{4 g^{2}}\left(\int d^{2} \theta W^{\alpha} W_{\alpha}+\text { c.c. }\right) \\
W^{\alpha} & =-\frac{1}{4} D \bar{D} D_{\alpha} V,
\end{aligned}
$$

where the $\Phi_{I}$ are charged chiral superfields and and $V$ is the vector superfield. The bosonic part is then the following:

$$
\begin{aligned}
\mathcal{L}_{K} & =-\frac{1}{4 g^{2}} F_{g}^{\mu \nu} F_{g \mu \nu}+g_{I \bar{J}} \nabla_{\mu} \phi^{I} \bar{\nabla}^{\mu} \phi^{\bar{J}}+\frac{g^{2}}{2}\left(\partial_{\left|\phi_{I}\right|^{2}} K\left(\left|\phi_{I}\right|^{2}\right)\left|\phi_{I}\right|^{2}-\zeta\right)^{2}, \\
g_{I \bar{J}} & =\partial_{\phi_{I} \bar{\phi}_{\bar{J}}} K\left(\left|\phi_{I}\right|^{2}\right)
\end{aligned}
$$

One can then employ a Bogomol'nyi completion:

$$
\begin{aligned}
\mathcal{E}_{K} & =\frac{1}{2 g^{2}}\left[F_{g 12}-g^{2}\left(\partial_{\left|\phi_{I}\right|^{2}} K\left(\left|\phi_{I}\right|^{2}\right)\left|\phi_{I}\right|^{2}-\zeta\right)\right]^{2}+4 g_{I \bar{J}} \nabla_{z} \phi^{I} \bar{\nabla}_{\bar{z}} \bar{\phi}^{\bar{J}}+ \\
& -\zeta F_{g 12}+\epsilon_{i j} \partial_{i} \mathcal{N}_{j} . \\
\mathcal{N}_{j} & =\frac{i}{2} \partial_{\left|\phi_{I}\right|^{2}} K\left(\left|\phi_{I}\right|^{2}\right) \phi^{I} \nabla_{j} \bar{\phi}^{\bar{I}}+\text { c.c. }
\end{aligned}
$$

As usual, vortex equations are given by imposing the vanishing of the squares in the first line of the expression above:

$$
\begin{aligned}
F_{g 12} & =g^{2}\left(\partial_{\left|\phi_{I}\right|^{2}} K\left(\left|\phi_{I}\right|^{2}\right)\left|\phi_{I}\right|^{2}-\zeta\right) \\
\nabla_{g \bar{z}} \phi_{I} & =0,
\end{aligned}
$$

where the second follows since the metric $g_{I J}$ is assumed to be positive definite.

\section{References}

[1] N. Seiberg and E. Witten, "Electric - magnetic duality, monopole condensation, and confinement in N=2 supersymmetric Yang-Mills theory", Nucl.Phys. B426, 19 (1994), hep-th/9407087. • N. Seiberg and E. Witten, "Monopoles, duality and chiral symmetry breaking in N=2 supersymmetric QCD", Nucl. Phys. B431, 484 (1994), hep-th/9408099.

[2] N. Dorey, T. J. Hollowood and D. Tong, "The BPS spectra of gauge theories in two and four dimensions", JHEP 9905, 006 (1999), hep-th/9902134. • N. Dorey, "The BPS spectra of two-dimensional supersymmetric gauge theories with twisted mass terms",

JHEP 9811, 005 (1998), hep-th/9806056. • Bolokhov, Pavel A. and Shifman, Mikhail and Yung, Alexei, "BPS Spectrum of Supersymmetric CP(N-1) Theory with Zn Twisted Masses", 1104.5241. 
[3] A. M. Polyakov and A. A. Belavin, "Metastable States of Two-Dimensional Isotropic Ferromagnets", JETP Lett. 22, 245 (1975).

[4] A. Belavin, A. M. Polyakov, A. Schwartz and Y. Tyupkin, "Pseudoparticle Solutions of the Yang-Mills Equations", Phys.Lett. B59, 85 (1975).

[5] N. S. Manton and P. Sutcliffe, "Topological solitons", Cambridge, UK: Univ. Pr. (2004) 493 p.

[6] T. Vachaspati and A. Achucarro, "Semilocal cosmic strings", Phys. Rev. D44, 3067 (1991).

[7] A. Achucarro and T. Vachaspati, "Semilocal and electroweak strings", Phys. Rept. 327, 347 (2000), hep-ph/9904229.

[8] M. Hindmarsh, "Existence and stability of semilocal strings", Phys. Rev. Lett. 68, 1263 (1992).

[9] M. Hindmarsh, "Semilocal topological defects", Nucl. Phys. B392, 461 (1993), hep-ph/9206229.

[10] J. Preskill, "Semilocal defects", Phys. Rev. D46, 4218 (1992), hep-ph/9206216.

[11] A. Belavin, V. Fateev, A. S. Schwarz and Y. Tyupkin, "Quantum Fluctuations Of Multi Instanton Solutions", Phys.Lett. B83, 317 (1979). • D. Diakonov and M. Maul, "On statistical mechanics of instantons in the $C P^{* *}(N(c)-1)$ model", Nucl.Phys. B571, 91 (2000), hep-th/9909078. - D. Son, M. A. Stephanov and A. Zhitnitsky, "Instanton interactions in dense matter QCD", Phys.Lett. B510, 167 (2001), hep-ph/0103099, • A. R. Zhitnitsky, "Confinement- deconfinement phase transition and fractional instanton quarks in dense matter", hep-ph/0601057.

[12] B. Collie and D. Tong, "The Partonic Nature of Instantons", JHEP 0908, 006 (2009), 0905.2267.

[13] M. Eto et al., "Fractional Vortices and Lumps", Phys. Rev. D80, 045018 (2009), 0905.3540.

[14] S. B. Gudnason, "Fractional and semi-local non-Abelian Chern-Simons vortices", Nucl.Phys. B840, 160 (2010), 1005.0557.

[15] A. A. Abrikosov, "On the Magnetic properties of superconductors of the second group", Sov. Phys. JETP 5, 1174 (1957). • H. B. Nielsen and P. Olesen, "Vortex-Line Models for Dual Strings", Nucl. Phys. B61, 45 (1973).

[16] B. Schroers, "Bogomolny solitons in a gauged O(3) sigma model", Phys.Lett. B356, 291 (1995), hep-th/9506004. • B. J. Schroers, "The Spectrum of Bogomol'nyi Solitons in Gauged Linear Sigma Models", Nucl. Phys. B475, 440 (1996), hep-th/9603101.

[17] J. M. Baptista, "Vortex equations in abelian gauged sigma-models", Commun. Math. Phys. 261, 161 (2006), math/0411517.

[18] J. Baptista, "A Topological gauged sigma-model", Adv.Theor.Math.Phys. 9, 1007 (2005), hep-th/0502152. • J. Baptista, "On the $L^{2}$-metric of vortex moduli spaces", Nucl.Phys. B844, 308 (2011), 1003.1296.

[19] D. J. Gross, "Meron Configurations in the Two-Dimensional O(3) Sigma Model", Nucl.Phys. B132, 439 (1978).

[20] J. Callan, Curtis G., R. F. Dashen and D. J. Gross, "A Mechanism for Quark Confinement", Phys.Lett. B66, 375 (1977). • J. Callan, Curtis G., R. F. Dashen and D. J. Gross, "A Theory of Hadronic Structure", Phys.Rev. D19, 1826 (1979). 
[21] A. Hanany and D. Tong, "Vortex strings and four-dimensional gauge dynamics", JHEP 0404, 066 (2004), hep-th/0403158.

[22] M. Shifman and A. Yung, "Non-Abelian string junctions as confined monopoles", Phys. Rev. D70, 045004 (2004), hep-th/0403149.

[23] M. Shifman and A. Yung, "Non-Abelian semilocal strings in $N=2$ supersymmetric QCD", Phys. Rev. D73, 125012 (2006), hep-th/0603134.

[24] M. Eto et al., "On the moduli space of semilocal strings and lumps", Phys. Rev. D76, 105002 (2007), 0704.2218.

[25] P. Koroteev, M. Shifman, W. Vinci and A. Yung, "Quantum Dynamics of Low-Energy Theory on Semilocal Non-Abelian Strings", Phys.Rev. D84, 065018 (2011), 1107.3779.

[26] M. T. K. Kasamatsu and M. Ueda, "Vortices in Multicomponet Bose-Einstein Condensates", Int. J. Mod. Phys. B19, 1835 (2005). • M. Eto, K. Kasamatsu, M. Nitta, H. Takeuchi and M. Tsubota, "Interaction of half-quantized vortices in two-component Bose-Einstein condensates", Phys.Rev. A83, 063603 (2011).

[27] E. Babaev, "Vortices carrying an arbitrary fraction of magnetic flux quantum in two-gap superconductors", Phys. Rev. Lett. 89, 067001 (2002), cond-mat/0111192. • E. Babaev, "Phase diagram of a planar two band superconductor: Condensation of vortices with fractional flux quantum and existence of a nonsuperconducting superfluid state in this system", Nucl.Phys. B686, 397 (2004), cond-mat/0201547.

[28] Y. Tanaka, "Phase Instability in Multi-band Superconductors", J.Phys.Soc.Jpn. 70, 2844 (2001), • Y. Tanaka, "Soliton in Two-Band Superconductor", Phys.Rev.Lett. 88, 017002 (2001). • J. Goryo, S. Soma and H. Matsukawa, "Deconfinement of vortices with continuously variable fractions of the unit flux quanta in two-gap superconductors ", Europhys. Lett. 80, 17002 (2007), cond-mat/0608015.

[29] B. Zumino, "Supersymmetry and Kahler Manifolds", Phys. Lett. B87, 203 (1979). • L. Alvarez-Gaume and D. Z. Freedman, "Geometrical Structure and Ultraviolet Finiteness in the Supersymmetric Sigma Model", Commun.Math.Phys. 80, 443 (1981).

[30] E. B. Bogomol'nyi, "Stability of Classical Solutions", Sov. J. Nucl. Phys. 24, 449 (1976).

[31] M. Prasad and C. M. Sommerfield, "An Exact Classical Solution for the 't Hooft Monopole and the Julia-Zee Dyon", Phys.Rev.Lett. 35, 760 (1975).

[32] E. Witten and D. I. Olive, "Supersymmetry Algebras That Include Topological Charges", Phys.Lett. B78, 97 (1978).

[33] J. D. Edelstein, C. Nunez and F. Schaposnik, "Supersymmetry and Bogomolny equations in the Abelian Higgs model", Phys. Lett. B329, 39 (1994), hep-th/9311055.

[34] A. Rebhan, P. van Nieuwenhuizen and R. Wimmer, "Nonvanishing quantum corrections to the mass and central charge of the $N=2$ vortex and BPS saturation", Nucl.Phys. B679, 382 (2004), hep-th/0307282.

[35] J. Bagger and E. Witten, "The Gauge Invariant Supersymmetric Nonlinear Sigma Model", Phys.Lett. B118, 103 (1982).

[36] P. Fayet and J. Iliopoulos, "Spontaneously Broken Supergauge Symmetries and Goldstone Spinors", Phys. Lett. B51, 461 (1974). 
[37] C. H. Taubes, "Arbitrary N: Vortex Solutions to the First Order Landau-Ginzburg Equations", Commun.Math.Phys. 72, 277 (1980) • G. W. Gibbons, M. E. Ortiz, F. Ruiz Ruiz and T. M. Samols, "Semilocal strings and monopoles", Nucl. Phys. B385, 127 (1992), hep-th/9203023

[38] Y. Isozumi, M. Nitta, K. Ohashi and N. Sakai, "All exact solutions of a 1/4 Bogomol'nyi-Prasad- Sommerfield equation", Phys. Rev. D71, 065018 (2005), hep-th/0405129. • M. Eto, Y. Isozumi, M. Nitta, K. Ohashi and N. Sakai, "Moduli space of non-Abelian vortices", Phys. Rev. Lett. 96, 161601 (2006), hep-th/0511088. • M. Eto, Y. Isozumi, M. Nitta, K. Ohashi and N. Sakai, "Solitons in the Higgs phase: The moduli matrix approach", J. Phys. A39, R315 (2006), hep-th/0602170.

[39] M. Nitta and W. Vinci, "Non-Abelian Monopoles in the Higgs Phase", Nucl.Phys. B848, 121 (2011), 1012.4057.

[40] N. S. Manton, "A Remark on the Scattering of BPS Monopoles", Phys. Lett. B110, 54 (1982)

[41] M. Eto et al., "Constructing Non-Abelian Vortices with Arbitrary Gauge Groups", Phys. Lett. B669, 98 (2008), 0802.1020. • M. Eto et al., "Non-Abelian Vortices in $S O(N)$ and USp(N) Gauge Theories", JHEP 0906, 004 (2009), 0903.4471.

[42] M. Shifman, W. Vinci and A. Yung, "Effective World-Sheet Theory for Non-Abelian Semilocal Strings in $N=2$ Supersymmetric QCD", 1104.2077, * Temporary entry *.

[43] N. Seiberg, "Exact results on the space of vacua of four-dimensional SUSY gauge theories", Phys. Rev. D49, 6857 (1994), hep-th/9402044. • P. C. Argyres, M. R. Plesser and N. Seiberg, "The Moduli Space of N=2 SUSY QCD and Duality in N=1 SUSY QCD", Nucl. Phys. B471, 159 (1996), hep-th/9603042

[44] M. Eto et al., "Universal reconnection of non-Abelian cosmic strings", Phys. Rev. Lett. 98, 091602 (2007), hep-th/0609214.

[45] M. Eto, Y. Isozumi, M. Nitta, K. Ohashi and N. Sakai, "Instantons in the Higgs phase", Phys. Rev. D72, 025011 (2005), hep-th/0412048.

[46] A. Hanany and D. Tong, "Vortices, instantons and branes", JHEP 0307, 037 (2003), hep-th/0306150. • R. Auzzi, S. Bolognesi, J. Evslin, K. Konishi and A. Yung, "Nonabelian superconductors: Vortices and confinement in $N=2$ SQCD", Nucl. Phys. B673, 187 (2003), hep-th/0307287.

[47] M. Shifman and A. Yung, "Supersymmetric Solitons and How They Help Us Understand Non-Abelian Gauge Theories", Rev. Mod. Phys. 79, 1139 (2007), hep-th/0703267. D. Tong, "TASI lectures on solitons", hep-th/0509216. • D. Tong, "Quantum Vortex Strings: A Review", 0809.5060.

[48] M. G. Alford, A. Schmitt, K. Rajagopal and T. Schafer, "Color superconductivity in dense quark matter", Rev.Mod.Phys. 80, 1455 (2008), 0709.4635.

[49] A. Balachandran, S. Digal and T. Matsuura, "Semi-superfluid strings in high density QCD", Phys.Rev. D73, 074009 (2006), hep-ph/0509276. - M. Eto and M. Nitta, "Color Magnetic Flux Tubes in Dense QCD", Phys.Rev. D80, 125007 (2009), 0907.1278.

[50] E. Nakano, M. Nitta and T. Matsuura, "Non-Abelian Strings in High Density QCD: Zero Modes and Interactions", Phys. Rev. D78, 045002 (2008), 0708.4096. • M. Eto, E. Nakano and M. Nitta, "Effective world-sheet theory of color magnetic flux tubes in dense QCD", Phys.Rev. D80, 125011 (2009), 0908.4470 
[51] R. Auzzi and S. Prem Kumar, "Quantum Phases of a Vortex String", Phys.Rev.Lett. 103, 231601 (2009), 0908.4278.

[52] V. Markov, A. Marshakov and A. Yung, "Non-Abelian vortices in $N=1^{*}$ gauge theory", Nucl. Phys. B709, 267 (2005), hep-th/0408235. 